\title{
El estado del arte en gobernanza de destinos turísticos
}

\author{
State of art in tourist destination governance
}

\section{Françoise Queiroz}

Universidad de Málaga, Facultad de CC. Económicas y Empresariales, Departamento de Economía y Administración de Empresas, Campus El Ejido, s/n; 29071, Málaga, España, franqueiroz@uma.es

\section{María-Ángeles Rastrollo-Horrillo}

Universidad de Málaga, Facultad de CC. Económicas y Empresariales, Departamento de Economía y Administración de Empresas, Campus El Ejido, s/n; 29071, Málaga, España, rastrollo@uma.es

\section{Resumen}

El objetivo de este trabajo es avanzar en la delimitación de las dimensiones relevantes para la gobernanza de destinos turísticos, a partir de una revisión de la literatura que incorpora los hallazgos que ha proporcionado la rica experiencia en asociaciones público-privadas en turismo. Partiendo de que la gobernanza implica el funcionamiento coordinado de las partes interesadas en el destino turístico (nivel estructural) para alcanzar objetivos compartidos (nivel intencional), nos proponemos investigar sobre cómo se identifica quiénes son las partes interesadas en turismo, cuáles son sus motivaciones, qué recursos y capacidades son determinantes de su participación en los procesos de gestión, con qué roles y qué instrumentos favorecen la participación y la resolución de problemas, especialmente los derivados de conflictos de intereses. La gobernanza encuentra su espacio de argumentación dentro del análisis sobre la participación de los agentes interesados para la consecución de metas compartidas en turismo. En tal sentido, la gobernanza se configura como un modelo de gobierno útil para identificar cómo poner en práctica un ejercicio efectivo de sostenibilidad en los destinos turísticos. El proceso de participación puede abarcar desde el diagnóstico de la situación y el establecimiento de los objetivos del destino, pasando por el diseño de la política turística, su implementación y la evaluación, considerando la transparencia y la rendición de cuentas para asegurar la confianza.

Palabras claves: Gobernanza, destinos turísticos, participación, transparencia, rendición de cuentas.

\begin{abstract}
The objective of this paper is to advance the delineation of the relevant dimensions of tourist destination governance from a literature review, which incorporates the experience in public-private tourism partnerships. Assuming that governance involves stakeholders coordination in the tourist destination (structural level) to achieve shared goals (intentional level), we propose to investigate on how the key tourism stakeholders are identified, what resources and capabilities are determinants to participate in management processes, what roles and instruments encouraging participation and problem solving, especially conflict of interest. The participation of the stakeholders is of paramount relevance to the theoretical framework for the tourist destination governance, in order to reflect on how to satisfy needs of all stakeholders and to allow them participation in tourism development process to achieve shared goals. In this way, governance is a useful model of government to identify how to put into in practice an effective exercise of sustainability in tourist destinations. Participation processes can involve the situational diagnosis of tourist destinations, including the design of product, implementation and evaluation of the tourism policy, taking into account transparency and accountability to ensure trust.
\end{abstract}

Keywords: Governance, tourist destinations, participation, transparency, accountability.

\section{Introducción}

La gobernanza se sitúa en el debate actual de la política y la investigación turística como un nuevo enfoque de gobierno que dé respuesta a la creciente complejidad de la gestión de destinos turísticos. En un contexto de preocupación por la sostenibilidad de los destinos cobra sentido reflexionar sobre cómo gestionarlos para satisfacer las necesidades de todos sus integrantes y con la participación de todos a la hora de diseñar, ejecutar y evaluar la política turística.

El análisis de la gobernanza en destinos turísticos se puede considerar tributario de los estudios sobre colaboración público-privada que tanto han interesado en las últimas décadas a los gestores e investigadores. Por ello, un primer paso para acercarnos al estado del arte en gobernanza de destinos turísticos es revisar los estudios sobre colaboración público - privada.

La colaboración público - privada tiene un amplio historial en la literatura y en la práctica turística a nivel internacional, pero la gobernanza turística es un fenómeno más reciente (Beritelli et al. 2007). La mayoría de las publicaciones se basan en análisis de casos, identificándose imprecisiones en el concepto y ambigüedades terminológicas que llevan a utilizar, por ejemplo, como sinónimos gobernanza, colaboración, cooperación, coordinación. Esto supone una especie de debilidad en el marco conceptual de la gobernanza de destinos turísticos, dejando espacios abiertos a la reflexión: desde la propia delimitación del concepto hasta las herramientas que permiten el ejercicio de esta nueva práctica política del gobierno del destino.

En esta línea el trabajo de Ruhanen, Scott, Ritchie, y Tkaczynski, (2010), analiza la literatura relevante sobre gobernanza en un intento de identificar sus dimensiones, y concluyen en la multidimensionalidad del concepto y por lo tanto, en la necesidad de explorar las dimensiones específicas de la gobernanza en turismo, considerando las características particulares de un destino. La Organización Mundial del Turismo (UNWTO, 2013) y Duran (2013), avanzan en la sistematización de las características de la gobernanza turística, proporcionando una definición y un conjunto de indicadores que, a modo de ejemplo, ayuden a medir la gobernanza en el sector turístico y a su aplicación práctica. 
El objetivo de este trabajo es avanzar en la delimitación de las dimensiones teóricas relevantes sobre gobernanza de destinos turísticos, a partir de una revisión de la literatura que incorpora los hallazgos que ha proporcionado la rica experiencia en asociaciones público-privadas. Partiendo de que la gobernanza implica el funcionamiento coordinado entre las partes interesadas en el destino turístico (nivel estructural) para alcanzar objetivos compartidos (nivel intencional) (Caffyn \& Jobbins, 2003; Duran, 2013; UNWTO, 2013), nos proponemos investigar sobre cómo se identifica quiénes son las partes interesadas en turismo, cuáles son sus motivaciones, qué recursos y capacidades son determinantes de su participación en los procesos de gestión, con qué roles y qué instrumentos favorecen la participación y la resolución de problemas, especialmente los derivados de conflictos de intereses.

\section{Antecedentes de la Gobernanza: la colaboración para la gestión del turismo}

Para los investigadores la decisión de colaborar es una variable dependiente y utilizando diferentes teorías han buscado las variables independientes que condicionarían la colaboración, así se han interesado por conocer cómo se relacionan los actores, en qué colaboran y qué tipo de alianzas establecen.

La decisión de colaborar en turismo ha encontrado explicación en una amplia variedad de enfoques teóricos: Greenwood (1993), Wang y Fesenmaier (2007) y Nordin y Svensson (2007) han utilizado la Teoría de la Dependencia de Recursos (Pfeffer \& Salancik, 1978) argumentando que los grupos de interés poseen recursos importantes a través de los cuales se controlan unos a otros. Autores como Beritelli et al.( 2007), Wang (2008) y Wang y Fesenmaier (2007) han elegido la Teoría de los Costes de Transacción (Williamson, 1975) para explicar que la decisión de colaborar en turismo puede encontrar su motivación en la necesidad de adquirir ventajas competitivas como reducir los costes de información y negociación. Otros autores han aplicado la Teoría de la Gestión Estratégica (Porter, 1990; Prahalad \& Hamel, 1990) en el estudio de la colaboración entre las partes interesadas en turismo, concluyendo en la exigencia de "complementariedad" entre los agentes para crear un producto completo y ofrecer una experiencia integral al turista (Pechlaner \& Tschurtschenthaler, 2003; Wang \& Fesenmaier, 2007; y Czernek, 2013). Por el contrario, Wang y Fesenmaier (2007) explican la colaboración a partir de la Teoría de Redes (Freeman, 1984; Wood \& Gray, 1991; Granovetter, 1985, Gulati,1998), argumentando que las relaciones entre los agentes se impulsan por reconocimiento, aceptación mutua y la búsqueda por intereses comunes. Por último, partiendo de la Teoría del Ciclo de Vida, Czernek (2013) y Santos, Ferreira y Costa (2014) identifican los factores endógenos que pueden determinar la necesidad de colaboración, en el sentido de incrementar la competitividad frente a la entrada de nuevos destinos turísticos competidores.

Aunque algunos autores como Hall (1999) y Fyall y Garrod (2005), hayan admitido diferencias entre los términos colaboración, coordinación y cooperación recurriendo al grado de institucionalización de los acuerdos, el resultado no ha sido fructuoso, y se puede considerar que la literatura de manera general ha aceptado los términos como sinónimos, aunque se admitan diferentes modos o grados de colaboración.

Las colaboraciones han sido caracterizadas de una parte, como maduras o inmaduras, abarcando desde acuerdos breves en torno a un problema común hasta representaciones altamente estructuradas determinadas por acuerdos jurídicos (Palmer \& Bejou, 1995; Selin \& Chavez, 1995); de otra parte, las colaboraciones se han distinguido por la existencia o no de un mediador (Fyall, Garrod \& Wang, 2012). En tal línea, la investigación de Fyall et al. (2012), resume tales características a partir del análisis de las tipologías de organizaciones turísticas del destino. De acuerdo con los autores, en presencia de organizaciones maduras, con alta especialización y experiencia en turismo, existe una tendencia hacia colaboraciones directas entre organizaciones. Tales colaboraciones se concretan sea en la creación de grupos formales, en los que las relaciones se establecen por acuerdos jurídicos bajo nombres como "partnership", consorcios o club del producto; ya sea por acuerdos breves o puntuales, como por ejemplo, eventos específicos o desarrollo de pagina web. Por el contrario, en contextos de "empresas débiles" o "menos organizadas", así como en difíciles condiciones económicas, institucionales y ambientales, la presencia de un mediador entre las partes interesadas puede ser crucial, en el sentido de compensar la falta de poder y la poca influencia sobre los recursos de los agentes individuales. Sin embargo, la colaboración mediada no es fácil de implementar.

Según la literatura, variables como el comportamiento cooperativo y la reputación condicionan el proceso de colaboración. El comportamiento cooperativo se refiere a las preferencias y conexiones interpersonales de los actores (Beritelli, 2011). La reputación se relaciona con la fuerza y el respeto del mediador (Palmer \& Bejou, 1995; Connelly, 2007; Lemmetyinen, 2010) como podría ser la presencia de un empresario reconocido en el territorio (Strobl \& Peters, 2013). En tal línea, la reputación del mediador, puede compensar la falta de poder de los actores más débiles y permitirles un mejor posicionamiento en la toma de decisiones, es decir, al adoptar la etiqueta o la marca de otra organización conocida por medio de la colaboración, los actores más débiles logran comprensión y legitimidad (Zapata \& Hall, 2012).

En la literatura también se aprecia el interés por establecer los factores que determinarían el éxito de la colaboración para la gestión del destino. Son numerosos los factores críticos del éxito de las colaboraciones identificados: la disponibilidad de personal preparado, la existencia de objetivos a largo plazo, la claridad en la delimitación de los roles y de los procesos, la existencia de sistemas de monitoreo de los objetivos (Knowles, 2000); el grado de compromiso y la capacidad de consolidar la colaboración como modelo de negocio, la confianza y capacidad de asunción de riesgo conjuntos (Hwang, 2001; Nordin \& Svensson, 2007); la historia del destino, las asimetrías de poder, la interdependencia (Beritelli et al., 2007) o la 
inclusión de todas las partes interesadas (D’Angella \& Gob , 2009; Bregoli, 2013).

En ello, se inicia el debate sobre los modelos de gestión que favorecen el éxito de los destinos. De una parte se argumentan las bondades de los modelos empresariales para implementar estrategias de competitividad en un entorno de fuerte competencia internacional (Beritelli, Bieger \& Laesser 2007; Beritelli \& Laesser, 2011; Strobl \& Peters, 2013; Volgger \& Pechlaner, 2014). De otra parte se posicionan los autores que critican la sostenibilidad de las estrategias empresariales en el desarrollo del turismo local, al hacer caso omiso a los problemas locales y a las necesidades de los residentes del destino (Caffyn \& Jobbins, 2003; Velasco, 2007; Dinica, 2009; Beaumont \& Dredge, 2010; Dredge \& Whitford, 2011; Moscardo, 2011; Hultman \& Hall, 2012). Estos últimos autores defienden la idea de una mayor participación de los residentes y de otros grupos de interés, en cuanto legítimas partes interesadas. Vemos pues, que se reclama una mayor incorporación de todas las partes con incidencia en el destino turístico, y para avanzar en la incorporación de estos otros actores, en la gestión del destino turístico, se empieza a hacer uso del concepto de gobernanza.

\section{La gobernanza en la literatura sobre gestión de destinos turísticos}

En la literatura del inicio de los años 90 se identificaban básicamente dos grupos de actores potenciales como partes interesadas en la gestión del turismo (Palmer \& Bejou, 1995; Greenwood, 1993): el grupo de los intereses económicos (sector privado) y el grupo de los intereses públicos (representada por el organismo público de turismo, como secretarias, departamentos, etc.). Posteriormente, se amplían las partes interesadas, por ejemplo, en la investigación de Caffyn y Jobbins (2003) sobre la gestión integrada en zonas costeras se incorporan los usuarios locales, los científicos y las organizaciones no gubernamentales. De hecho, las investigaciones a partir del año 2000, se han centrado en discutir sobre la identificación de las partes interesadas para la gestión del destino, y sobre su participacón.

El concepto de gobernanza en los estudios de gestión viene introducido sobre todo en el análisis de la participación. Así encontramos estudios que argumentan sobre la participación de los residentes y de los grupos de interés público, como las ONGs y asociaciones comunitarias. Tales estudios se interesan por la participación de estos últimos (residentes y asociaciones) en diferentes contextos: en el desarrollo del turismo patrimonial (Aas, Ladkin \& Fletcher, 2005; McKercher, Ho \& du Cros, 2005; Garrod, Fyall, Leask \& Reid, 2012; Wang \& Bramwell, 2012) en la planificación del turismo (Paskaleva-Shapira, 2003; Jamal, 2004; Velasco, 2007; Mordue, 2007; Loulanski \& Loulanski, 2011; Moscardo, 2011; Dredge \& Whitford, 2011; Wan, 2013; Soares, Emmendoerfer \& Pereira, 2013) o en las asociaciones público-privadas (Hall, 1999; Velasco, 2007; Erkus-Öztürk \& Eraydın (2010); Zapata \& Hall, 2012).

En conclusión, esos autores verificaron que hay diferentes grados de participación entre los grupos de interés.
Además, algunas partes interesadas, aunque sean relevantes, no pueden intervenir en el proceso de toma de decisiones para "hacer valer" sus intereses. En tal línea, el poder es una variable que permea la discusión sobre participación. Quién tiene el poder determina las reglas y establece restricciones para la participación de algunas partes interesadas; pudiendo delegar el poder a otros. Se sostiene que las colaboraciones entre los sectores público y privado en turismo excluyeron a grupos de interés más amplios, como los residentes y los grupos de interés público, sobre todo en la toma de decisiones (Dredge \& Whitford, 2011; Wan, 2013). Claramente, el "empoderamiento" de estos últimos, es decir, el derecho a participar y la legitimidad en la toma de decisiones hacia la equidad de poder, no es condición fácil de ponerse en práctica. Eagles (2009) explica que la equidad entre las partes depende de la capacidad del sistema para proporcionar el acceso a la participación. Tejada, Santos y Guzmán (2011) sugieren que el principio de igualdad entre los participantes, como por ejemplo, el mismo nivel de especialización de los tour operadores, favorece el equilibrio de poder. Además, la complejidad del tema aumenta en la medida que hay que considerar la existencia de diferentes fuentes de poder controladas por las diferentes partes interesadas, como el poder de la autoridad, el poder de la acción social, el poder legítimo, el poder de las relaciones, el poder de la opinión pública, el poder experto (Beaumont \& Dredge, 2010).

Considerando tales dificultades, la gobernanza es propuesta como corriente teórica que establece mecanismos que permiten la participación de todas las partes interesadas que "implican" o son "implicadas" por el turismo, la inclusión de los intereses más amplios, la prevención del abuso de poder, y el aumento de la capacidad de respuesta en informar y explicitar las acciones llevadas a cabo (Hall, 1999, 2011; Caffyn \& Jobbins, 2003; Jamal, 2004; Hollinshead, 2009; Eagles, 2009; Dredge \& Whitford, 2011; Hultman \& Hall, 2012).

La literatura sobre gobernanza aplicada a destinos turísticos ha iniciado discusiones sobre "buen gobierno" y "mejores prácticas" a partir de análisis de casos en lugares y acontecimientos de interés turístico, como en los parques naturales (Jamal; 2004; Eagles, 2009), eventos (Dredge \& Whitford, 2011), zonas costeras (Caffyn \& Jobins, 2003; Wesley \& Pforr, 2010) y ciudades (Paskaleva-Shapira, 2003; 2005). Tales estudios han tenido diferentes objetos de investigación, como por ejemplo: los estudios en turismo sostenible y gestión integrada; los intereses y la participación en los procesos de colaboración; la gestión de las asociaciones; los desafíos y oportunidades asociados con este ideal teórico y práctico; la interacción entre poder y política en el sistema de desarrollo turístico; y la manera en que los diferentes niveles de la administración pública facilitan (o no) la mediación de las cuestiones relacionadas con la sostenibilidad. La tabla 1 sintetiza los estudios relevantes que introducen el concepto de gobernanza en el turismo. 
Tabla 1 - Estudios relevantes seleccionados sobre gobernanza en turismo

\begin{tabular}{|c|c|c|c|c|}
\hline Autor & Estudio & Marco Teórico & Metodología & Resultado \\
\hline $\begin{array}{l}\text { Caffyn \& Jobins } \\
(2003)\end{array}$ & $\begin{array}{l}\text { Exploran los elementos } \\
\text { que fomentan o } \\
\text { restringen el enfoque } \\
\text { holístico, } \\
\text { interdependiente e } \\
\text { inclusivo requerido por } \\
\text { el turismo sostenible y } \\
\text { la gestión integrada de } \\
\text { las costas }\end{array}$ & $\begin{array}{l}\text { Utiliza el marco teórico } \\
\text { de Kooiman ; análisis } \\
\text { de interacciones entre } \\
\text { los stakeholders }\end{array}$ & $\begin{array}{l}\text { Entrevista semi- } \\
\text { estructurada a los grupos } \\
\text { de interés, datos } \\
\text { secundarios de las } \\
\text { agencias estatales, como } \\
\text { los planes regionales, } \\
\text { estrategias de turismo y } \\
\text { los datos demográficos, } \\
\text { los resultados de } \\
\text { investigación de los } \\
\text { socios del proyecto MECO }\end{array}$ & $\begin{array}{l}\text { Elementos que } \\
\text { restringen: estructuras } \\
\text { rígidas de gobierno, } \\
\text { centralización, poco } \\
\text { intercambio de } \\
\text { información, falta de } \\
\text { análisis integrado y } \\
\text { poca participación. } \\
\text { Elementos que } \\
\text { fomentan: procesos } \\
\text { más abiertos, } \\
\text { integrados e inclusivos, } \\
\text { los cambios de enfoque } \\
\text { de los sistemas de } \\
\text { gobierno }\end{array}$ \\
\hline Jamal (2004) & $\begin{array}{l}\text { Examina los intereses y } \\
\text { la participación en los } \\
\text { procesos de } \\
\text { colaboración en áreas } \\
\text { protegidas }\end{array}$ & $\begin{array}{l}\text { Revisión de la } \\
\text { literatura sobre los } \\
\text { procesos de la gestión } \\
\text { de conflictos entre } \\
\text { múlti - interesados }\end{array}$ & $\begin{array}{l}\text { Estudio de caso, análisis } \\
\text { de la representación de } \\
\text { intereses y participación }\end{array}$ & $\begin{array}{l}\text { Concluye en la } \\
\text { necesidad de una } \\
\text { reevaluación crítica del } \\
\text { concepto de } \\
\text { participación; y la } \\
\text { necesidad de reforzar } \\
\text { la representación de } \\
\text { intereses del sector, } \\
\text { diseñando un proceso } \\
\text { que permita el diálogo } \\
\text { constructivo }\end{array}$ \\
\hline $\begin{array}{l}\text { Nordin \& } \\
\text { Svensson } \\
(2007)\end{array}$ & $\begin{array}{l}\text { Examinan la relación } \\
\text { entre la estructura de } \\
\text { gobernanza y el } \\
\text { desarrollo de destinos } \\
\text { turísticos, centrándose } \\
\text { en las relaciones } \\
\text { público- privadas } \\
\text { (gobierno local y sector } \\
\text { turístico) y la } \\
\text { dependencia de } \\
\text { recursos de redes } \\
\text { formales y informales }\end{array}$ & $\begin{array}{l}\text { Marco conceptual de } \\
\text { Gobernanza de los } \\
\text { Destinos turísticos }\end{array}$ & $\begin{array}{l}\text { Estudio de caso de la } \\
\text { primera estación de esquí } \\
\text { de Suecia; uso de la teoría } \\
\text { de la dependencia de } \\
\text { recursos }\end{array}$ & $\begin{array}{l}\text { Sugiere que el impacto } \\
\text { positivo depende de las } \\
\text { relaciones público- } \\
\text { privadas basadas en la } \\
\text { confianza, la asunción } \\
\text { de riesgos conjunta, las } \\
\text { estructuras informales } \\
\text { y los consensos } \\
\text { estratégicos }\end{array}$ \\
\hline Velasco (2007) & $\begin{array}{l}\text { Indaga si la idea de } \\
\text { gobernanza permite } \\
\text { ilustrar posibles vías de } \\
\text { desarrollo para } \\
\text { fortalecer una gestión } \\
\text { de destinos más eficaz } \\
\text { e inclusiva }\end{array}$ & $\begin{array}{l}\text { Utiliza el concepto de } \\
\text { gobernanza para } \\
\text { reflexionar sobre las } \\
\text { dificultades que el } \\
\text { gobierno local enfrenta } \\
\text { en la gestión de } \\
\text { destinos }\end{array}$ & $\begin{array}{l}\text { Reflexión teórica sobre la } \\
\text { idea de gobernabilidad } \\
\text { del turismo }\end{array}$ & $\begin{array}{l}\text { Sugiere cuatro } \\
\text { dimensiones de la } \\
\text { gobernanza del } \\
\text { turismo: } \\
\text { Existencia de un plan } \\
\text { estratégico, actores } \\
\text { comprometidos en la } \\
\text { gestión del turismo a } \\
\text { nivel local, capacidades } \\
\text { institucionales } \\
\text { efectivas participación } \\
\text { de los ciudadanos en el } \\
\text { proceso }\end{array}$ \\
\hline $\begin{array}{l}\text { Beritelli, Bieger } \\
\text { \& Laesser } \\
(2007)\end{array}$ & $\begin{array}{l}\text { Cuestionan los modelos } \\
\text { adecuados de } \\
\text { Gobernanza en } \\
\text { Turismo }\end{array}$ & $\begin{array}{l}\text { Marco en micro teorías } \\
\text { de "corporate } \\
\text { governance" }\end{array}$ & $\begin{array}{l}\text { Estudio de casos; casos } \\
\text { múltiples con } 12 \text { destinos } \\
\text { turísticos en los Alpes } \\
\text { suizos }\end{array}$ & $\begin{array}{l}\text { Identifican seis } \\
\text { dimensiones } \\
\text { relacionadas con la } \\
\text { gobernanza } \\
\text { corporativa: (1) los } \\
\text { costes de transacción, } \\
\text { (2) las asimetrías de } \\
\text { poder, (3) la } \\
\text { interdependencia, (4) } \\
\text { la confianza / control, } \\
\text { (5) el conocimiento y } \\
\text { (6) conexiones } \\
\text { informales, personales }\end{array}$ \\
\hline Eagles (2009) & $\begin{array}{l}\text { Utiliza los criterios de } \\
\text { gobernanza para } \\
\text { evaluar la gestión de } \\
\text { las asociaciones de ocio } \\
\text { y turismo asociados a } \\
\text { los parques y a las } \\
\text { áreas protegidas }\end{array}$ & $\begin{array}{l}\text { Modelos de } \\
\text { Gobernanza en parques } \\
\text { naturales }\end{array}$ & $\begin{array}{l}\text { Establece criterios de } \\
\text { gobernanza y aplican a } \\
\text { los modelos } \\
\text { seleccionados }\end{array}$ & $\begin{array}{l}\text { Concluye que hay } \\
\text { mayor énfasis en : } \\
\text { eficiencia, } \\
\text { participación, visión } \\
\text { estratégica y capacidad } \\
\text { de respuesta. Menor } \\
\text { énfasis: rendición de } \\
\text { cuentas y la } \\
\text { transparencia. }\end{array}$ \\
\hline
\end{tabular}




\begin{tabular}{|c|c|c|c|c|}
\hline Autor & Estudio & Marco Teórico & Metodología & Resultado \\
\hline $\begin{array}{l}\text { Wesley \& Pforr } \\
(2010)\end{array}$ & $\begin{array}{l}\text { Analizan la gobernanza } \\
\text { para lograr una mayor } \\
\text { comprensión de los } \\
\text { desafíos y } \\
\text { oportunidades } \\
\text { asociados con este } \\
\text { ideal teórico y práctico; } \\
\text { analizan la interacción } \\
\text { entre poder y política } \\
\text { en el sistema de } \\
\text { desarrollo turístico }\end{array}$ & Gobernanza y turismo & $\begin{array}{l}\text { Caso de estudio Western } \\
\text { Australia - costero }\end{array}$ & $\begin{array}{l}\text { Sugiere la falta de } \\
\text { medios apropiados } \\
\text { para la gobernanza, y la } \\
\text { influencia de las } \\
\text { relaciones de poder } \\
\text { para la participación de } \\
\text { los interesados }\end{array}$ \\
\hline $\begin{array}{l}\text { Beaumont \& } \\
\text { Dredge }(2010)\end{array}$ & $\begin{array}{l}\text { Investigan la manera } \\
\text { en que las diferentes } \\
\text { redes de gobernanza de } \\
\text { turismo locales operan } \\
\text { y los efectos de ello en } \\
\text { materia de política } \\
\text { turística local }\end{array}$ & $\begin{array}{l}\text { Revisión de la } \\
\text { literatura sobre las } \\
\text { dimensiones de la } \\
\text { gobernanza }\end{array}$ & $\begin{array}{l}\text { Estudio de caso } \\
\text { comparativo de tres } \\
\text { redes de gobernanza } \\
\text { locales }\end{array}$ & $\begin{array}{l}\text { Identifica tensiones en } \\
\text { el ámbito de (1) la } \\
\text { eficiencia en } \\
\text { comparación con la } \\
\text { inclusión, (2) la } \\
\text { legitimidad interna } \\
\text { frente externo y (3) la } \\
\text { flexibilidad frente a la } \\
\text { estabilidad }\end{array}$ \\
\hline $\begin{array}{l}\text { Robterson } \\
\text { (2011) }\end{array}$ & $\begin{array}{l}\text { Analizan el sistema de } \\
\text { gobernanza } \\
\text { colaborativa }\end{array}$ & $\begin{array}{l}\text { Revisión teórica de la } \\
\text { gobernanza } \\
\text { colaborativa(redes) } \\
\text { aplicada a la gestión del } \\
\text { turismo }\end{array}$ & $\begin{array}{l}\text { Estudio de caso: } \\
\text { Redeturis en Rio de } \\
\text { Janeiro }\end{array}$ & $\begin{array}{l}\text { El impacto de una red } \\
\text { colaborativa en el } \\
\text { turismo depende de } \\
\text { que sea reconocida } \\
\text { como un legitimo } \\
\text { stakeholder y del } \\
\text { establecimiento básico } \\
\text { de confianza }\end{array}$ \\
\hline $\begin{array}{l}\text { Gill \& Willian } \\
\text { (2011) }\end{array}$ & $\begin{array}{l}\text { Se interesaron en } \\
\text { conocer se el enfoque } \\
\text { empresarial de gestión } \\
\text { aplicado a la } \\
\text { sostenibilidad integral } \\
\text { imponen restricciones } \\
\text { a la adopción de } \\
\text { caminos alternativos de } \\
\text { gobernanza }\end{array}$ & $\begin{array}{l}\text { Literatura sobre } \\
\text { gobernanza }\end{array}$ & $\begin{array}{l}\text { Énfasis en los factores } \\
\text { críticos que afectan a la } \\
\text { evolución de los enfoques } \\
\text { de gobernanza a partir de } \\
\text { Estudio de caso }\end{array}$ & $\begin{array}{l}\text { La incertidumbre no } \\
\text { está relacionada con } \\
\text { los mecanismos reales } \\
\text { de democracia } \\
\text { participativa, sino más } \\
\text { bien con cuestiones } \\
\text { tales como las agendas } \\
\text { políticas;las estrategias } \\
\text { competitivas } \\
\text { empresariales y las } \\
\text { relaciones de poder } \\
\text { que subyacen en la } \\
\text { toma de decisiones }\end{array}$ \\
\hline $\begin{array}{l}\text { Dredge \& } \\
\text { Whitford } \\
\text { (2011) }\end{array}$ & $\begin{array}{l}\text { Analizan las } \\
\text { características del } \\
\text { espacio público en la } \\
\text { puesta en escena un } \\
\text { gran evento; exploran } \\
\text { la manera en que las } \\
\text { diferentes esferas } \\
\text { públicas facilitan (o no) } \\
\text { la mediación de las } \\
\text { cuestiones } \\
\text { relacionadas con la } \\
\text { sostenibilidad }\end{array}$ & $\begin{array}{l}\text { Consideran la "esfera } \\
\text { pública" - como espacio } \\
\text { de diálogo y de } \\
\text { participación, y su rol } \\
\text { en los cambios } \\
\text { sociológicos y políticos } \\
\text { más amplios }\end{array}$ & $\begin{array}{l}\text { Estudio de caso; la } \\
\text { gobernanza aplicada al } \\
\text { Campeonato Mundial de } \\
\text { Rally australiano (AWRC) } \\
\text { de } 2009\end{array}$ & $\begin{array}{l}\text { Sugiere que la gestión } \\
\text { empresarial de la } \\
\text { agencia de eventos y el } \\
\text { consiguiente } \\
\text { distanciamiento del } \\
\text { gobierno, minimiza los } \\
\text { intereses comunitarios } \\
\text { y dificulta su } \\
\text { participación en la } \\
\text { toma de decisiones en } \\
\text { cuanto al uso de los } \\
\text { recursos (rendición de } \\
\text { cuentas y } \\
\text { transparencia) }\end{array}$ \\
\hline Hall (2011) & $\begin{array}{l}\text { Tipología de las } \\
\text { distintas formas de } \\
\text { gobernanza del turismo } \\
\text { en cuanto instrumentos } \\
\text { de intervención política }\end{array}$ & $\begin{array}{l}\text { Desarrollo de una } \\
\text { tipología de los marcos } \\
\text { conceptuales de la } \\
\text { gestión pública. }\end{array}$ & $\begin{array}{l}\text { Utiliza las teorías } \\
\text { contractuales y la } \\
\text { tipología de los diferentes } \\
\text { marcos de gobernanza }\end{array}$ & $\begin{array}{l}\text { Identifica cuatro } \\
\text { marcos o tipos de } \\
\text { gobernabilidad en la } \\
\text { literatura de gobierno: } \\
\text { jerarquías, mercados, } \\
\text { redes y comunidades }\end{array}$ \\
\hline $\begin{array}{l}\text { Pechlaner, } \\
\text { Volgger \& } \\
\text { Herntrei (2012) }\end{array}$ & $\begin{array}{l}\text { Las relaciones entre el } \\
\text { gobernanza } \\
\text { empresarial de las } \\
\text { Destination } \\
\text { Management } \\
\text { Organization y la } \\
\text { gobernanza del destino }\end{array}$ & $\begin{array}{l}\text { Base teórica sobre } \\
\text { gestión empresarial }\end{array}$ & $\begin{array}{l}\text { Estudio de caso; sur del } \\
\text { Tirol del Eppan-Kaltern- } \\
\text { Tramin (Italia) }\end{array}$ & $\begin{array}{l}\text { Concluyen que la } \\
\text { gobernanza está } \\
\text { fuertemente ligada a la } \\
\text { aceptación de la DMO } \\
\text { por parte de los actores } \\
\text { del destino; la relación } \\
\text { positiva entre la } \\
\text { gobernanza } \\
\text { empresarial de las } \\
\text { DMOs se caracteriza } \\
\text { por una amplia } \\
\text { participación de los } \\
\text { stakeholders, así como } \\
\text { una forma eficiente de }\end{array}$ \\
\hline
\end{tabular}




\begin{tabular}{|c|c|c|c|c|}
\hline Autor & Estudio & Marco Teórico & Metodología & Resultado \\
\hline & & & & $\begin{array}{l}\text { trabajar y una } \\
\text { actuación visible y una } \\
\text { gobernanza de destino } \\
\text { asociada con un alto } \\
\text { nivel de cooperación }\end{array}$ \\
\hline $\begin{array}{l}\text { Hultman \& Hall } \\
(2012)\end{array}$ & $\begin{array}{l}\text { Analizan los destinos y } \\
\text { la interacción social de } \\
\text { los actores que } \\
\text { participan en la gestión }\end{array}$ & $\begin{array}{l}\text { Se utilizan el concepto } \\
\text { de gobernanza para } \\
\text { aclarar las relaciones } \\
\text { de entre las partes } \\
\text { interesadas }\end{array}$ & $\begin{array}{l}\text { Estudio de caso; cuatro } \\
\text { proyectos de desarrollo } \\
\text { de economía de destinos } \\
\text { diferentes en el sur de } \\
\text { Suecia }\end{array}$ & $\begin{array}{l}\text { Concluyen que la } \\
\text { adopción de una } \\
\text { perspectiva de la } \\
\text { gobernanza permiten } \\
\text { una comprensión } \\
\text { diferente de la gestión } \\
\text { del turismo y de los } \\
\text { discursos de marketing } \\
\text { de servicios }\end{array}$ \\
\hline Duran (2013) & $\begin{array}{l}\text { Analiza las } \\
\text { dimensiones de la } \\
\text { gobernanza para la } \\
\text { construcción de } \\
\text { indicadores }\end{array}$ & $\begin{array}{l}\text { Marco teórico de la } \\
\text { gobernanza en turismo }\end{array}$ & Conceptual & $\begin{array}{l}\text { Identifica dos } \\
\text { dimensiones. } \\
\text { La dimensión } \\
\text { capacidad del gobierno } \\
\text { directivo: poderes y } \\
\text { recursos disponibles } \\
\text { (para la colaboración y } \\
\text { coordinación de la } \\
\text { cooperación de una } \\
\text { manera co- } \\
\text { responsable, } \\
\text { transparente y } \\
\text { responsable); } \\
\text { La dimensión eficiencia } \\
\text { directiva: ejercicio } \\
\text { eficiente (de la } \\
\text { coordinación, la } \\
\text { colaboración y la } \\
\text { cooperación basada en } \\
\text { la corresponsabilidad, } \\
\text { la transparencia y la } \\
\text { rendición de cuentas) }\end{array}$ \\
\hline
\end{tabular}

Fuente: Elaboración propia.

Partiendo de las recomendaciones del Banco Mundial sobre mecanismos para favorecer la gobernanza, Eagles (2009) y Beaumont y Dredge, (2010) se interesan por el uso de instrumentos de transparencia, de participación y de rendición de cuentas de los agentes implicados en la gestión del turismo, ya sean administraciones públicas, organizaciones empresariales o la ciudadanía. Otros autores, si bien no hacen mención explícita al concepto de gobernanza sí coinciden en la necesidad de crear normativas que favorezcan la participación amplia, en que se incluye la ciudadanía, en la toma de decisiones turísticas y la rendición de cuentas. La transparencia y la rendición de cuentas son claves para el establecimiento de la credibilidad y la confianza (Gill \& Willian, 2011; Beaumont \& Dredge, 2010) que favorecen la participación en la obtención de metas compartidas.

En relación a la transparencia, se argumenta la importancia de los mecanismos de comunicación para garantizar el derecho a la información (divulgación completa de todas las actividades, checks anuales, balance) y se materializan en paginas web, foros, consejos, juntas, reuniones abiertas (Caffyn \& Jobbins, 2003; Paskaleva-Shapira, 2005; Beaumont \& Dredge, 2010). La rendición de cuentas se facilita por el establecimiento de mecanismos de control continuos, como por ejemplo el voto, instrumentos de responsabilidad social y consultas populares (Wang \& Bramwell, 2012), y mediante el uso de sanciones para aquellos que no respectan las reglas, a través de organizaciones con autoridad, como los consejos de control o las agencias de control (Gill \& Willian, 2011; Eagles, 2009; Beaumont \& Dredge, 2010; Robterson, 2011; Dredge \& Whitford, 2011; Wesley \& Pforr, 2010; Duran, 2013; UNWTO, 2013).
Las investigaciones, sin embargo, observaron que hay factores que pueden dificultar la participación de determinados agentes por falta de tiempo, problemas para similar la información, no acceso a las tecnologías de la información y comunicación, o escasez de recursos financieros (Caffyn \& Jobbins, 2003; Aas et al., 2005; Moscardo, 2011; Soares et al., 2013).

Los estudios mencionados también buscan las variables decisivas para el éxito del proceso de gobernanza en turismo y mencionan: el compromiso efectivo de los organismos interesados públicos y privados, así como de la comunidad residente, la legitimidad para participar, los objetivos de interés colectivo, la implementación de herramientas de gestión y de comunicación constructiva, la aceptación de la diversidad, la búsqueda de equidad y el desarrollo del conocimiento y aprendizaje (Beaumont \& Dredge, 2010; Duran, 2013; UNWTO, 2013).

Como se puede observar, se presenta toda una panoplia de variables en las que se entrelazan características de los participantes, con instrumentos de comunicacióncoordinación-concertación y de rendición de cuentas que faciliten el proceso de acción conjunta para la obtención de metas compartidas.

\section{Conclusiones}

La revisión de literatura ha permitido identificar los antecedentes de la gobernanza en turismo y su introducción en cuanto concepto teórico. La gobernanza encuentra su espacio de argumentación dentro del análisis sobre la 
participación de los agentes interesados para asegurar la consecución de metas compartidas en turismo. En tal sentido, la gobernanza se configura como un modelo de gobierno útil para identificar cómo poner en práctica un ejercicio efectivo de sostenibilidad en los destinos turísticos. Para ello, se ha investigado quiénes son las partes interesadas en la gobernanza, qué recursos y capacidades son determinantes para la participación de las partes interesadas en los procesos de gestión, qué roles y cuáles son los instrumentos que favorecen la participación y la resolución de problemas. Tales aspectos se pueden agrupar en 3 dimensiones teóricas: ¿Quiénes?, ¿Qué? y ¿Cómo? detallados en la tabla 2.

Tabla 2 - Dimensiones teóricas para la Gobernanza en Destinos Turísticos

\begin{tabular}{|c|c|c|}
\hline Dimensiones & Delimitación & Factores claves \\
\hline ¿Quiénes? & $\begin{array}{l}\text { Identificación actores } \\
\text { Participación de la variedad de actores (sector } \\
\text { público, privado, otras organizaciones, } \\
\text { ciudadanía) }\end{array}$ & $\begin{array}{l}\text { Legitimidad y representatividad } \\
\text { Capacidad para motivar la participación de la } \\
\text { variedad de actores } \\
\text { Metas colectivas }\end{array}$ \\
\hline ¿Qué? & $\begin{array}{l}\text { Según las áreas de actividad (planificación, } \\
\text { diseño de producto, promoción y } \\
\text { comercialización) } \\
\text { Según la modalidad de participación en proceso } \\
\text { de decisiones (consulta, propone alternativas, } \\
\text { elige alternativas, ejecuta, evalúa) } \\
\text { Variable: en función de la actividad los roles de } \\
\text { los actores irán cambiando. }\end{array}$ & $\begin{array}{l}\text { Formación } \\
\text { Compromiso } \\
\text { Recursos financieros } \\
\text { Tiempo para participar } \\
\text { Recursos tecnológicos }\end{array}$ \\
\hline ¿Cómo? & $\begin{array}{l}\text { Transparencia y rendición de cuentas: } \\
\text { participación en el ciclo de la política turística } \\
\text { (identificación de la situación, diseño, } \\
\text { implementación y evaluación) }\end{array}$ & $\begin{array}{l}\text { Capacidad para hacer respetar las reglas } \\
\text { Mecanismos de control y de sanciones } \\
\text { Capacidad de rendición de cuentas } \\
\text { Instrumentos : redes y coordinación multinivel } \\
\text { (nacional, regional, local), DMO; } \\
\text { Web site, foros, consejos, juntas, planificación; } \\
\text { Aspectos normativos, incentivos } \\
\text { Agencias de control } \\
\text { Voto, consultas populares } \\
\text { Calendario anual de actividades }\end{array}$ \\
\hline & & \\
\hline
\end{tabular}

Fuente: Elaboración propia.

La identificación de los actores debe determinar las partes interesadas en el devenir del destino para garantizar la consecución de metas compartidas. El reto está en determinar las características de esos actores para encontrar el equilibrio entre las partes, y asegurar la participación efectiva de la variedad de actores dotándoles de legitimidad y representatividad.

Poner en práctica un ejercicio efectivo de participación de los actores implica poseer recursos y capacidades en materia del turismo, es decir, formación, recursos financieros, tiempo para participar, información y recursos tecnológicos compatibles con las áreas de actividad (planificación, diseño de producto, promoción y comercialización). En función de la actividad los roles de los actores irán cambiando, según el grado de participación en el proceso de toma de decisiones (consulta, proposición de alternativas, elección, ejecución, evaluación).

La transparencia en el proceso de gobernanza es fundamental para estimular las relaciones entre las partes interesadas al informar sobre las modalidades de participación, las normas, las reglas, las actividades y metas compartidas, como por ejemplo, modalidades para la presentación de proyectos y uso de los recursos financieros.

La rendición de cuentas garantiza el buen funcionamiento del proceso, el equilibrio de poder y el alcance de las metas compartidas, a través mecanismos de control continuos y de sanciones por no respeto a las reglas. Por lo tanto, transparencia y la rendición de cuentas aseguran la confianza en el proceso.

La Gobernanza se materializa en la existencia de instrumentos normativos, estructurales y organizativos, esos relacionados con la capacidad de gestión, como redes y coordinación multinivel (nacional, regional, local), DMO (Destination Management); instrumentos de comunicacióncoordinación-concertación (web site, foros, consejos, juntas, planificación, incentivo) y los instrumentos de responsabilidad social, consultas populares, voto $\mathrm{y}$ calendario anual de actividades; agencias de control y efectividad de los aspectos normativos.

El proceso de participación puede abarcar desde el diagnóstico de la situación y el establecimiento de los objetivos del destino, pasando por el diseño de la política turística, su implementación y la evaluación, considerando la transparencia y la rendición de cuentas para asegurar la confianza.

Por último, es importante puntualizar que las dimensiones teóricas identificadas en este estudio son de crucial relevancia por dos motivos. El primero porque ayuda a clarificar el concepto de gobernanza aplicado al turismo, y el segundo porque proporciona la base teórica para la construcción de indicadores que ayuden a una aplicación práctica de la gobernanza de los destinos turísticos. 


\section{Referencias}

Aas, C., Ladkin, A. \& Fletcher, J. (2005). Stakeholder collaboration and heritage management. Annals of Tourism Research, 32 (1), 28-48.

Beaumont, N. \& Dredge, D. (2010). Local tourism governance: a comparison of three network approaches. Journal of Sustainable Tourism, 18(1), 7-28.

Beritelli, P. (2011). Cooperation among prominent actors in a tourist destination. Annals of Tourism Research, 38(2), 607-629.

Beritelli, P. \& Laesser, C. (2011). Power dimensions and influence reputation in tourist destinations: empirical evidence from a network of actors and stakeholders. Tourism Management, 32, $1299-1309$

Beritelli, P., Bieger, T. \& Laesser, C. (2007). Destination governance: Using corporate governance theories as a foundation for effective destination management. Journal of Travel Research, 46, 96 - 107.

Bregoli, I. (2013). Effects of DMO coordination on destination brand identity: a mixed-method study on the city of Edinburgh. Journal of Travel Research, 52(2),212 -224.

Caffyn, A. \& Jobbins, G. (2003). Governance capacity and stakeholder interactions in the development and management of coastal tourism: Examples from Morocco and Tunisia. Journal of Sustainable Tourism, 11(2), 224-245.

Connelly, G. (2007). Testing govnance. A research agenda for exploring urban tourism competitiveness policy: the case of Liverpool 1980-2000. Tourism Geographies, 9(1), 84-114.

Czernek, K. (2013). Determinants of cooperation in a tourist region. Annals of Tourism Research, 40, 83-104.

D’Angella, F. \& Gob, M. F. (2009). Tale of two cities collaborative tourism marketing: towards a theory of destination stakeholder assessment. Tourism Management, 30, 429-440.

Dente, B. \& Coletti, P. (2011). Measuring governance in urban innovation. Local Government Studies, 37(1), 43-56.

Dinica, V. (2009). Governance for sustainable tourism: a comparison of international and dutch visions. Journal of Sustainable Tourism, 17(5), 583 - 603.

Dredge, D. \& Whitford, M. (2011). Event tourism governance and the public sphere. Journal of Sustainable Tourism, 19(4-5): 479-499.

Duran, C. (2013). Governance for the tourism sector and its measurement. UNWTO Statistics and TSA. Issue Paper Series STSA/IP/2013/01. Retrieve in 22 April 2013, http://statistics.unwto.org/en/content/papers.

Eagles, P. F. J. (2009). Governance of recreation and tourism and tourism partnerships in parks and protected areas. Journal of Sustainable Tourism, 17(2), 231-248.

Erkus-Öztürk, H. \& Eraydın, A. (2010). Environmental governance for sustainable tourism development: collaborative networks and organisation building in the Antalya tourism region. Tourism Management, 31, 113-124.

Freeman, R. E., \& McVea, J. (1984). A stakeholder approach to strategic management. Boston: Pitman

Fyall, A., Garrod, B. \& Wang, Y. (2012). Destination collaboration: A critical review of theoretical approaches to a multi-dimensional phenomenon. Journal of Destination Marketing \& Management, 1, $10-26$.

Fyall, A.,\&Garrod,B.(2005). Tourism marketing: A collaborative approach. Clevedon: Channel View Publications.

Garrod, B., Fyall, A, Leask, A. \& Reid, E. (2012). Engaging residents as stakeholders of the visitor attraction. Tourism Management, 33, $1159-1173$

Gill, M. A. \& Willian, W. P. (2011). Rethinking resort growth: understanding evolving governance strategies in Whistler. British Columbia. Journal of Sustainable Tourism, 19 (4-5), 629-648.

Granovetter, M. (1985). Economic action and social structure: the problem of embeddedness. American Journal of Sociology, 91(3), 481.

Greenwood, J. (1993). Business interest groups in tourism governance. Tourism Management, 335-348.

Gulati, R. (1995). Social structure and alliance formation patterns: A longitudinal analysis. Administrative Science Quarterly, 40, 619-653.

Hall, C. M. (1999). Rethinking collaboration and partnership: a public policy perspective. Journal of Sustainable Tourism, 7(3), 274 - 289.
Hall, C. M.(2011). A typology of governance and its implications for tourism policy analysis. Journal of Sustainable Tourism, 19(4/5), 437-457.

Hollinshead, K. (2009). Turism state cultural production: the remaking of Nova Scotia. Tourism Geographies, 11(4), 526-545.

Hultman, J. \& Hall, C. M. (2012). Tourism place-Making governance of locality in Sweden. Annals of Tourism Research, 39(2), 547-570.

Hwang, W. (2001). Conceptualizing inter-regional partnership for tourism promotion: In case of the East Asia inter-regional tourism forum (EATOF). Asia Pacific Journal of Tourism Research, 6(1), 26-36.

Jamal,T. (2004). Conflict in natural area destinations: a critique of representation and interest participatory processes. Tourism Geographies, 6(3), 352-379.

Knowles, T. (2000). Performance of tourism partnerships: a focus on York Marcjanna M. Augustyn. Tourism Management, 21, 341- 351.

Lemmetyinen, A. (2010). The role of the DMO in creating value in EU-funded tourism projects. Scandinavian. Journal of Hospitality and Tourism, 10(2), 129-152.

Loulanski, T. \& Loulanski, V. (2011). The sustainable integration of cultural heritage and tourism: a meta study. Journal of Sustainable Tourism, 19(7), 837-862.

McKercher, B, Ho, S. Y. \& du Cros, H. (2005). Relationships between tourism and cultural heritage management. Tourism Management, 26(4), $539-548$.

Mordue, T. (2007). Tourism, urban governance and public space. Leisure Studies, 26(4), 447-462.

Moscardo, G. (2011). Exploring social representations of tourism planning: issues for governance. Journal of Sustainable Tourism, 19(4/5), 423-436

Nordin, S. \& Svensson, B. (2007). Innovative destination governance: the swedish ski resort of Åre. Entrepreneurship and Innovation, 8(1), 53-66.

Palmer, A. \& Bejou, D. (1995). Tourism destination marketing. Annals of Tourism Research, 22(3), 616-629.

Paskaleva-Shapira, K. (2003). EU sut-governance project , City of tomorrow and cultural heritage. Final Report. R\&D Project of key Action. Retrieve in 22 April 2013, http://www.itas.kit.edu/pub/v/2003/pask03a.pdf.

Paskaleva-Shapira, K. (2005). Innovative partnerships for effective governance of sustainable urban tourism. R\&D Project of key Action 4, The city of tomorrow and cultural heritage.

Pechlaner, H. \& Tschurtschenthaler, P. (2003). Tourism policy, tourism organisations and change management in Alpine regions and destinations: a european perspective. Current Issues in Tourism, 6(6), 508-539.

Pechlaner, H., Volgger, M. \& Herntrei, M. (2012). Destination management organizations as interface between destination governance and corporate governance. Anatolia, 23(2), 151-168.

Pfeffer, J., and G. Salancik.(1978). The external control of organizations: a resource-dependence perspective. New York: Harper and Row.

Porter,M. E. (1990). The competitive advantage of nations. New York, NY: The Free Press.

Prahalad CK, Hamel G. 1990. The core competence of the corporation. Harvard Business Review, 68(3): 79-91.

Robterson, J. P. (2011). An assessment of collaborative governance in a network for sustainable tourism: the case of RedeTuris. International Journal of Public Administration, 34(5), 279-290.

Ruhanen, L., Scott, N., Ritchie, B. \& Tkaczynski, A. (2010). Governance: a review and synthesis of the literature. Tourism Review, 65(4), 4-16.

Santos, M; Ferreira, A.M. \& Costa, C. (2014). Influential factors in the competitiveness of mature tourism destinations. Tourism \& Management Studies, 10(1), 73-81.

Selin, S. \& Chavez, D. (1995). Developing an evolutionary tourism partnership model West Virginia University, USA. Annals Of Tourism Research, 22(4), 844-856.

Soares, E; Emmendoerfer, M \& Monteiro, L. (2013). Gestão pública no turismo e o desenvolvimento de destinos turísticos em um estado da Federação Brasileira: uma análise do planejamento estratégico do turismo em Minas Gerais (2007-2010). Tourism \& 
Management Studies, 9(2), 50-56.

Strobl, A. \& Peters, M. (2013). Entrepreneurial reputation in destination networks. Annals of Tourism Research, 40, 59-82.

Tejada, P., Santos, F. J. \& Guzmán, J. (2011). Applicability of global value chain analysis to tourism: issues of governance and upgrading. The Service of Industries Journal, 31(10), 1643- 1647.

Velasco, G. M. (2007). Gestión de destinos: ¿Gobernabilidad del turismo o gobernanza del destino? Ministerio de educación y ciencia de España. Plan nacional de I+D+I (2004- 2007).

Volgger, M. \& Pechlaner, H. (2014). Requirements for destination management organizations in destination governance: Understanding DMO success. Tourism Management, 41, 64 -75.

Wan, K. P. Y. (2013). A comparison of the governance of tourism planning in the two special administrative regions (SARs) of China e Hong Kong and Macao. Tourism Management, 36, 164-177.

Wang, Y. (2008). Collaborative destination marketing: understanding the dynamic process. Journal of Travel Research 47(2), 151-166.

Wang, Y. \& Bramwell, B. (2012). Heritage protection and tourism development priorities in Hangzhou, China: A political economy and governance perspective. Tourism Management, 33, 988-998.

Wang, Y. \& Fesenmaier, R. D. (2007). Collaborative destination marketing: A case study of Elkhart county, Indiana. Tourism Management, 28, 863-875.

Wesley , A. \& Pfoor, C. (2010) . The governance of coastal tourism:unravelling the layers of complexity at Smiths Beach, Westearn Australia. Journal of Sustanaible Tourism, 18(6), 773 -792.

Williamson, O.E. (1975). Markets and hierarchies: analysis and antitrustimplications. The Free Press, New York.

Wood, D.J., and B. Gray.(1991). Towards a comprehensive theory of collaboration. The Journal of Applied Behavioral Science 27:139-162.

World Tourism Organization (2013). Sustainable tourism governance and management in coastal areas of Africa. UNWTO, Madrid.

Zapata, J. M. \& Hall, C. M. (2012). Public-private collaboration in the tourism sector: balancing legitimacy and effectiveness in local tourism partnerships. The spanish case. Journal of Policy Research in Tourism, Leisure and Events, 4(1), 61-83.

Recibido: 30.06.2014

Aceptado: 04.12.2014 